\title{
RECOVERIES OF BLACK-CROWNED NIGHT HERONS BANDED IN SASKATCHEWAN
}

by C. Stuart Houston, 863 University Drive, Saskatoon

Early banding of Black-crowned Night Herons in Saskatchewan yielded a very high number of recoveries, compared to later banding. The late George H. Lang banded this species almost yearly from 1924 to 1939 at "Dry Lake", the easternmost of the two Strawberry Lakes south of Indian Head. His biggest years were 1925, when he banded $110 ; 1926$, when he banded 149; and 1932 when he banded 138. Of the first 609 banded up to the end of 1932 , there were 24 recoveries, a rate of $3.9 \%$, but from the last 250 banded there was not a single recovery! My personal banding of 183 night herons with four recoveries, represents a recovery rate of $2.2 \%$. Note how the earlier recoveries include many birds shot, whereas in mor recent years such non-game bird seem less likely to be shot illegally.

The oldest Saskatchewan bird wa nearly nine years old when it wa electrocuted and found hanging fron a power pole.

It is of interest that in 1964 , Bo Nero found Black-crowned Nigh Herons nesting between the tw Strawberry Lakes. I banded 44 tha year, but to date have had no recov eries from them. Mr. Lang's daughte reported that their colony was $n$ longer present at "Dry Lake" in th early 1940's.

\section{RECOVERIES OF BLACK-CROWNED NIGHT HERONS BANDED IN SASK ATCHEW AN}

(in the following table, 532-1040 means $53^{\circ} 20^{\prime}$ north and $104^{\circ} 00^{\prime}$ west)

Banded by Judge I. A. M. Patrick at Foam Lake, Sask. (514-1033):

Banded July 4/29. Shot hunting season/34 (5 yrs.) near Quinton, Sask. (512-1042).

Banded July 4/29. Shot Oct. 20/29 (direct) in Texas (273-0993).

Banded by George H. Lang at Strawberry Lakes (502-1034): (859 banded)

Banded June 18/24.

Electric pole, May 8/33 (9 yrs.) 30 mi. N. Borger, Texas (360-1012).

Banded June 18/24. Shot Dec. 1/24 (direct) Hidalgo Co., Texas (26x-097x).

Banded June 18/24. Found dead early May/26 (2 yrs.) Dunseith, N.D. (484-1000).

Banded June 23/24. Injured Nov. 27/24 (direct) Deerfield, Florida (261-0800) Banded July $4 / 24$.

Banded July $4 / 24$.

Banded July $4 / 24$.

Banded July $15 / 25$.

Banded July $15 / 25$.

Banded July $15 / 25$.

Banded July 15/25.

Banded July 15/25.

Banded June 12/26.

Banded June 12/26.

Banded June 12/26.

Banded June 26/26.

Banded June 26/26.

Banded June 26/26.

Banded June $11 / 27$.

Found dead mid-May/27 (3 yrs.) Oakes, N.D. (460-0980) Shot Sept. 21/24 (direct) Valley City, N.D. (465-0975). Shat Sept. 21/24 (direct) in state of Vera Cruz, Mexico. Found dead Oct. 11/25 (direct) Adair, Sask. (502-1031). Found dead Mar. 9/32 (7 yrs.) Edenwold, Sask. (503-1041 Found dead July/25 (direct) Francis, Sask. (500-1035). Found dead Oct. 16/32 (7yrs.) Carman, Ill. (404-0910). Shot Oct. $30 / 25$ (direct) Carroll Co., Ark. (36x-093x). Shot Sept. 15/28 (2 yrs.) Penzance, Sask. (510-1052).

Shot early Oct./28 (2 yrs.) Calhoun, Georgia. (342-0845) Shot Oct. 19/26 (direct) Throckmorton, Texas (331-0991) Shot Oct. 8/26 (direct) St. Joseph, Missouri (394-0945). Shot Oct. 9/26 (direct) St. Joseph, Missouri (394-0945). Shot Nov. 1/26 (direct) Fort Smith, Okla. (352-0943). Found dead June 22/28 (1 yr.) Strawberry Lakes, Sask. (502-1034). 
anded June 24/28. Shot Oct. 7/28 (direct) Valley City, Ill. (394-0903).

nded June 24/28. Shot April 17/34 (6 yrs.) Central Butte, Sask. (504-1063).

anded June 23/29. Found dead Aug. 22/29 (direct). Ft. Qu'Appelle, Sask.

(502-1034).

nded June 30/29. Shot Sept. 25/29 (direct) Warwick, N.D. (475-0984).

nded July 11/31. Trapped and released Oct. 8/31 (direct) McPherson, Kans. (382-0973).

nded July 11/31. Caught in fish net, Oct. 12/31 (direct) Eastland, Texas (322-0984).

nded July 9/32. Caught by fish hook, May 1/37 (5 yrs.) Marlin, Texas (311-0965).

nded July 9/32. Found dead Oct. 26/32 (direct) Montevideo, Minn.

(445-0954).

nded July 9/32. Caught in trap Nov. 20/32 (direct) Romeo, Ill. (413-0880).

anded July 9/32. Found dead July/42 (10 yrs.) Weyburn, Sask. (493-1035).

anded July $9 / 32 \quad$ Killed by auto Oct. 23/32 (direct) Sarasota, Florida

(273-0821).

anded July 12/32. Found dead Oot. 13/32 (direct) Bordulac, N.D. (472-0985).

anded by C. Stuart Houston, Yorkton, Sask. (511-1023 and 510-1022)

(183 banded) :

anded June 18/44. Found dead before Sept. 16/52 (8 yrs.) Wichita, Kans. (375-0972).

anded July 2/44. Found dead Oct. 12/47 (3 yrs.) Welda, Kans. (381-0951). anded July 25/46. Found dead Oct. 10/47 (1 yr.) Morris Ill. (412-0882). anded June 9/55. Killed by oil before Oct. 18/56 (1 yr.) Asher, Okla. (345-0965).

anded by Fred G. Bard, at Davidson, Sask. (511-1055) : anded July 6/36 Found dead Nov. 7/36 (direct) in Minnesota (455-0955).

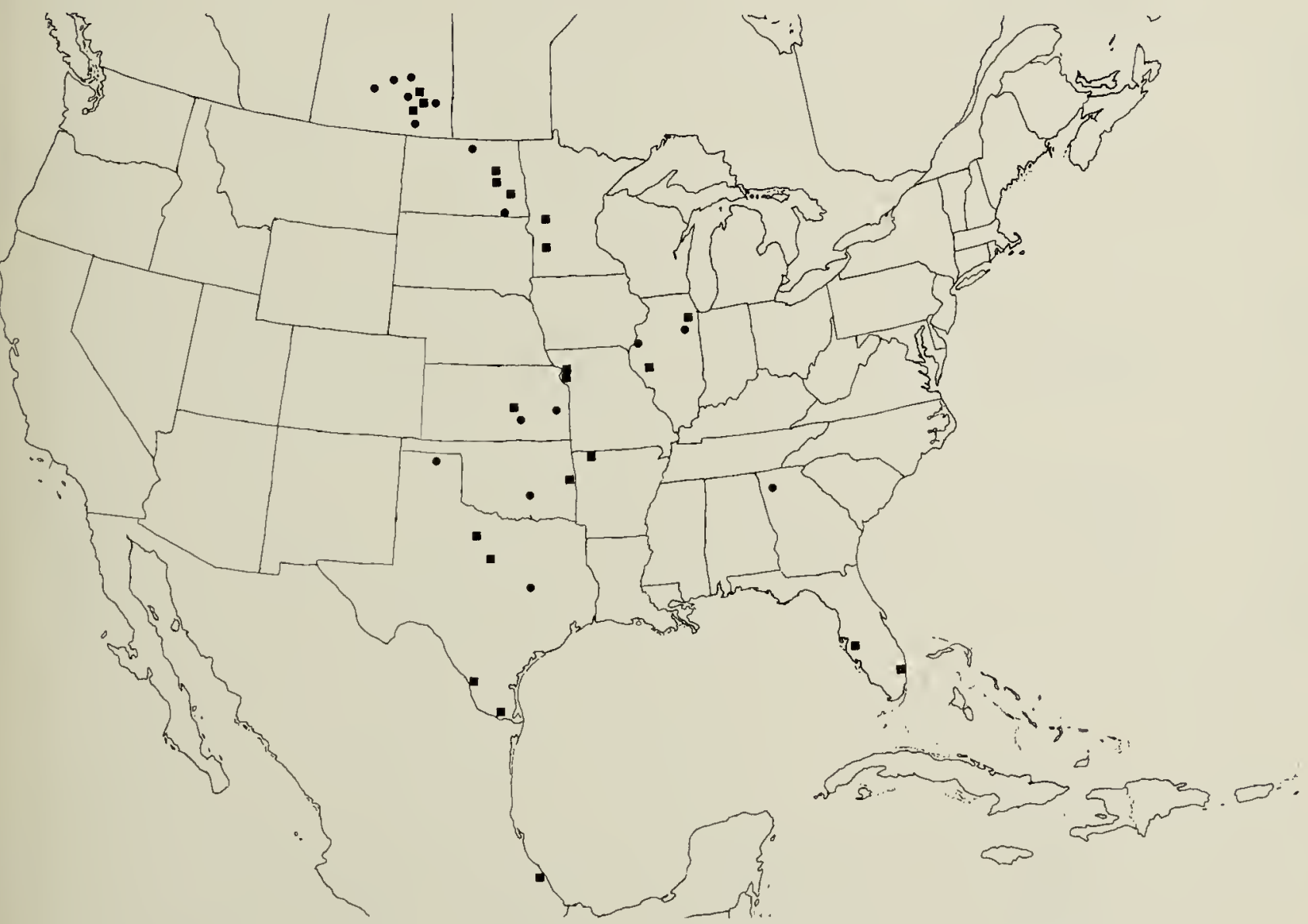

Recoveries of Black-crowned Night Herons banded in Saskatchewan

Note: Squares represent direct recoveries (same year). Circles represent subsequent years. 


\begin{tabular}{|c|c|c|c|c|c|c|c|c|c|c|c|c|}
\hline $\begin{array}{l}\text { SH'N uooteyses } \\
\text { NOOI } \forall X S \forall S\end{array}$ & $\frac{\infty}{\frac{0}{\alpha}}$ & $\begin{array}{l} \pm \\
\frac{0}{\alpha}\end{array}$ & $\begin{array}{l}\text { N } \\
Q \\
Q\end{array}$ & $\frac{1}{\frac{0}{4}}$ & $\begin{array}{l}\text { 尺 } \\
\text { Q } \\
\text { \& }\end{array}$ & $\frac{m}{\frac{m}{\alpha}}$ & $\begin{array}{l}\text { N } \\
\stackrel{2}{<}\end{array}$ & $\begin{array}{l}\bar{N} \\
\frac{0}{\alpha}\end{array}$ & $\begin{array}{l}\stackrel{2}{2} \\
\text { 之े }\end{array}$ & & $\begin{array}{l}\bar{N} \\
\stackrel{Q}{\alpha}\end{array}$ & $\frac{\infty}{\sum^{x}}$ \\
\hline $\begin{array}{c}\text { S.H'N eu!bay } \\
\forall N I S \exists Y\end{array}$ & $\frac{\square}{\frac{Z}{2}}$ & $\begin{array}{l}\infty \\
\frac{Q}{4}\end{array}$ & $\begin{array}{l}\infty \\
\frac{Q}{<}\end{array}$ & $\begin{array}{l}\infty \\
\stackrel{Q}{<}\end{array}$ & $\begin{array}{l}\stackrel{\rho}{e} \\
\stackrel{2}{\Sigma}\end{array}$ & $\stackrel{\text { N }}{\stackrel{\text { L }}{\Sigma}}$ & $\begin{array}{l}\stackrel{\nabla}{ } \\
\text { Q }\end{array}$ & $\frac{1}{\frac{0}{4}}$ & $\begin{array}{l}N \\
\simeq\end{array}$ & & $\begin{array}{l}= \\
\frac{0}{8}\end{array}$ & $\begin{array}{l}\hat{=} \\
\hat{\Sigma}\end{array}$ \\
\hline 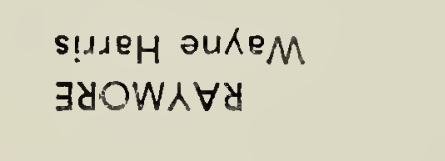 & $\frac{m}{\frac{m}{\alpha}}$ & $\begin{array}{l}\infty \\
\frac{2}{\alpha}\end{array}$ & $\frac{\bar{a}}{\frac{\alpha}{\alpha}}$ & $\frac{\bar{a}}{\frac{2}{4}}$ & $\begin{array}{l}\frac{0}{2} \\
\frac{2}{\alpha}\end{array}$ & $\frac{0}{\frac{0}{\alpha}}$ & $\frac{\infty}{\frac{0}{\alpha}}$ & $\begin{array}{l}\text { N } \\
\stackrel{0}{\alpha}\end{array}$ & $\begin{array}{l}\stackrel{\text { N }}{N} \\
\stackrel{\vec{\Sigma}}{\vec{z}}\end{array}$ & $\begin{array}{l}\nabla \\
\subsetneq\end{array}$ & $\begin{array}{l}\overline{0} \\
\frac{0}{4}\end{array}$ & $\begin{array}{l}\stackrel{N}{N} \\
\stackrel{2}{\Sigma}\end{array}$ \\
\hline 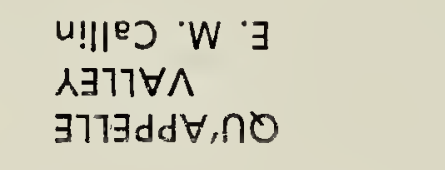 & $\stackrel{N}{N}$ & $\begin{array}{l}\infty \\
2 \\
\alpha\end{array}$ & $\frac{\bar{a}}{\frac{0}{4}}$ & $\frac{\bar{a}}{\frac{\alpha}{\alpha}}$ & $\begin{array}{l}\stackrel{D}{N} \\
\sum\end{array}$ & $\begin{array}{l}\stackrel{\mathrm{N}}{\mathrm{N}} \\
\stackrel{\Sigma}{\Sigma}\end{array}$ & $\frac{N}{\frac{2}{\alpha}}$ & $\frac{0}{\frac{0}{4}}$ & $\begin{array}{l}\stackrel{2}{2} \\
\stackrel{2}{\Sigma}\end{array}$ & $\begin{array}{l}\stackrel{\text { N }}{N} \\
\stackrel{2}{\Sigma}\end{array}$ & $\frac{\infty}{\frac{\infty}{\alpha}}$ & $\begin{array}{l}\stackrel{P}{2} \\
\stackrel{\lambda}{\Sigma}\end{array}$ \\
\hline 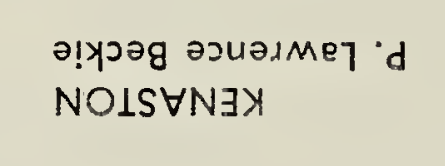 & ${ }^{\nabla}$ & $\begin{array}{l}\text { ลे } \\
0 \\
\alpha\end{array}$ & $\frac{O}{\frac{0}{4}}$ & $\frac{m}{\frac{m}{\alpha}}$ & $\begin{array}{l}\stackrel{P}{N} \\
\stackrel{\llcorner}{\Sigma}\end{array}$ & $\frac{1}{\frac{0}{4}}$ & $\stackrel{\stackrel{N}{N}}{\stackrel{\gtrless}{\Sigma}}$ & $\begin{array}{l}\text { N } \\
\text { Q } \\
\end{array}$ & & & $\begin{array}{l}\stackrel{Z}{N} \\
\frac{Q}{4}\end{array}$ & $\begin{array}{l}\stackrel{\Delta}{N} \\
\underset{\Sigma}{\Sigma}\end{array}$ \\
\hline 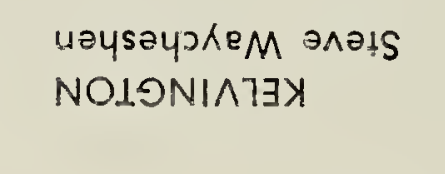 & & $\begin{array}{l}0 \\
0 \\
\alpha\end{array}$ & $\begin{array}{l}\stackrel{\nabla}{N} \\
0 \\
0\end{array}$ & $\begin{array}{l}\stackrel{+}{N} \\
\frac{0}{\alpha}\end{array}$ & $\frac{n}{\frac{0}{2}}$ & $\frac{\square}{2}$ & $\bar{\Sigma}$ & $\frac{\infty}{2}$ & $\stackrel{\substack{N \\
\grave{\Sigma}}}{\stackrel{2}{2}}$ & $\hat{a}^{\lambda}$ & $\begin{array}{l}1 \\
\frac{2}{4}\end{array}$ & 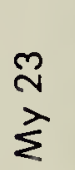 \\
\hline 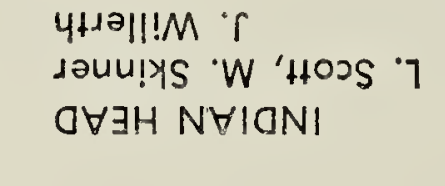 & $\frac{a}{4}$ & $\begin{array}{l}0 \\
\vdots \\
\Sigma\end{array}$ & $\begin{array}{l}\stackrel{2}{N} \\
\stackrel{\llcorner}{\Sigma}\end{array}$ & $\begin{array}{l}\infty \\
\frac{2}{8}\end{array}$ & $\begin{array}{l}\stackrel{\infty}{N} \\
\stackrel{2}{\Sigma}\end{array}$ & $\frac{0}{<}$ & $\stackrel{a}{\Sigma}$ & $\frac{a}{4}$ & $\stackrel{0}{5}$ & 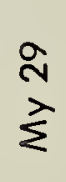 & $\frac{0}{\frac{0}{2}}$ & $\begin{array}{l}\bar{N} \\
\bar{\Sigma}\end{array}$ \\
\hline $\begin{array}{l}\text { HelX } \Rightarrow \text { 'y } \\
\text { X्रS }\end{array}$ & $\stackrel{2}{\Sigma}$ & $\begin{array}{l}\overline{2} \\
\frac{2}{4}\end{array}$ & $\frac{1}{2}$ & $\frac{0}{2}$ & $\begin{array}{l}\text { N } \\
\stackrel{\Sigma}{\llcorner}\end{array}$ & $\begin{array}{l}\stackrel{2}{N} \\
\stackrel{2}{<}\end{array}$ & & $\stackrel{0}{\Sigma}$ & & & $\frac{N}{\frac{N}{4}}$ & $\begin{array}{l}\stackrel{\infty}{N} \\
\sum\end{array}$ \\
\hline 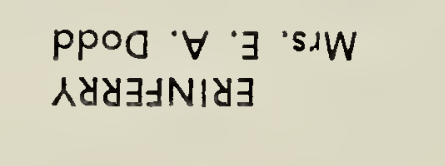 & & $\begin{array}{l}0 \\
\frac{0}{<} \\
<\end{array}$ & $\begin{array}{l}\text { N } \\
\frac{Q}{<}\end{array}$ & $\begin{array}{l}\text { N } \\
\frac{0}{<}\end{array}$ & $\frac{m}{\frac{m}{\alpha}}$ & $\frac{0}{\frac{0}{2}}$ & $\frac{0}{\grave{k}}$ & $\frac{\infty}{\frac{\infty}{\alpha}}$ & $\stackrel{\circ}{\stackrel{N}{\Sigma}}$ & & & $\begin{array}{l} \\
\end{array}$ \\
\hline 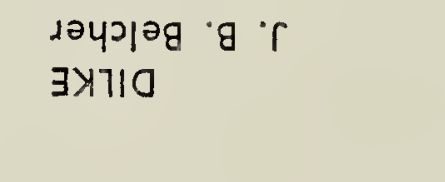 & $\begin{array}{l}\text { ลิ } \\
\text { Q }\end{array}$ & $\begin{array}{l}\circ \\
\text { ह } \\
\sum\end{array}$ & $\frac{1}{\frac{0}{4}}$ & $=$ & $\begin{array}{l}\stackrel{N}{\grave{\Sigma}} \\
\dot{\Sigma}\end{array}$ & $\bar{z}$ & & $\begin{array}{l}\text { N } \\
\text { रे }\end{array}$ & & & $\begin{array}{l}\bar{N} \\
\dot{\alpha} \\
\dot{\alpha}\end{array}$ & $\begin{array}{l}\text { N } \\
\underset{\Sigma}{\Sigma}\end{array}$ \\
\hline 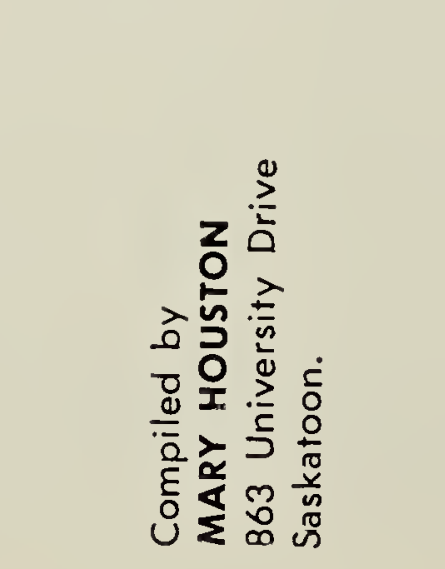 & 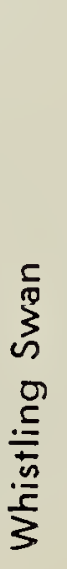 & $\begin{array}{l}0 \\
\tilde{0} \\
0 \\
0 \\
0 \\
0 \\
0 \\
0 \\
0 \\
0 \\
0\end{array}$ & $\begin{array}{l}\frac{0}{0} \\
\frac{0}{00} \\
\overline{10}\end{array}$ & $\begin{array}{l}\stackrel{\bar{\pi}}{ \pm} \\
\stackrel{ \pm}{0}\end{array}$ & 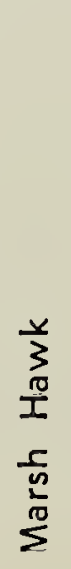 & $\frac{\frac{d}{d}}{\frac{d}{0}}$ & 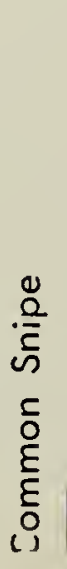 & 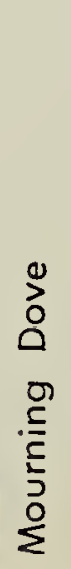 & 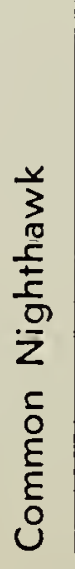 & 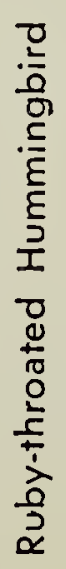 & 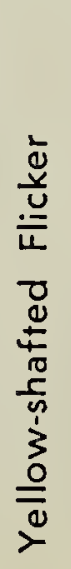 & 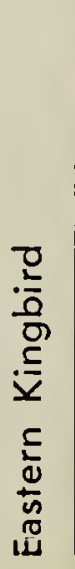 \\
\hline
\end{tabular}




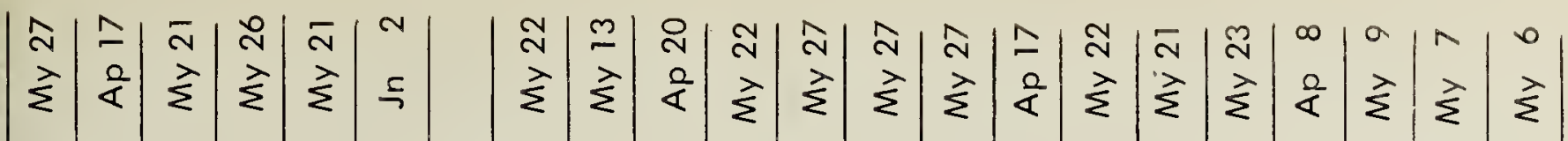

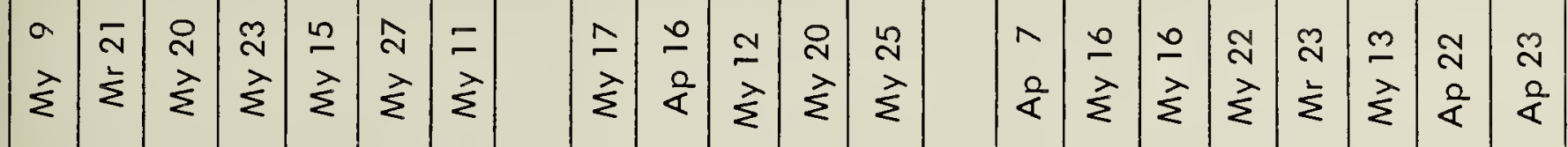

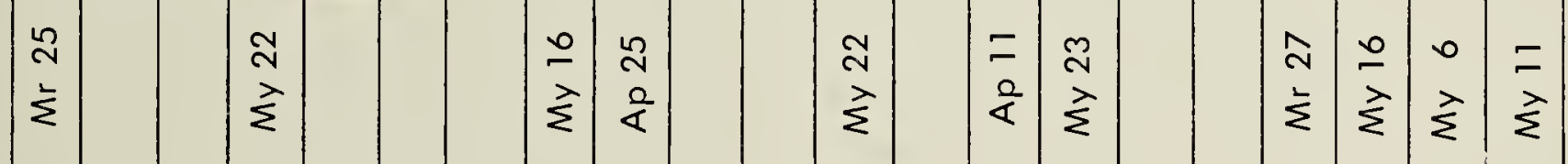

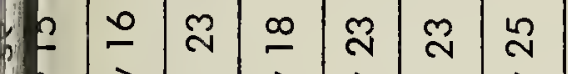

\begin{tabular}{|c|c|c|c|c|c|c|c|c|c|c|c|c|c|c|}
\hline 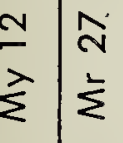 & $\mid$\begin{tabular}{l}
0 \\
\} $\\
{\text { 之े }}$ & $\frac{0}{\grave{\Sigma}}$ & $\begin{array}{l}\infty \\
N \\
\Sigma\end{array}$ & & $\begin{array}{l}0 \\
\frac{0}{4}\end{array}$ & & $\mid \begin{array}{l}0 \\
\grave{\Sigma}\end{array}$ & & $\begin{array}{l}\stackrel{\infty}{N} \\
⿱ 亠 乂\end{array}$ & & $\mid \begin{array}{l}\infty \\
2 \\
\&\end{array}$ & $\frac{0}{3}$ & $\frac{m}{\sum}$ & $\frac{0}{2}$ \\
\hline $\begin{array}{l}\bar{N} \\
\grave{\Sigma}\end{array}$ & & $\begin{array}{l}\bar{N} \\
\bar{\Sigma}\end{array}$ & & $\begin{array}{l}- \\
\subsetneq\end{array}$ & & $\begin{array}{l}\stackrel{J}{N} \\
\stackrel{\lambda}{\Sigma}\end{array}$ & $\mid \begin{array}{l}n \\
0 \\
8\end{array}$ & $\mid \begin{array}{l}n \\
N \\
\Sigma\end{array}$ & & 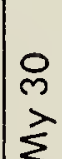 & $\mid \begin{array}{l}0 \\
N \\
\Sigma\end{array}$ & & & 之े \\
\hline
\end{tabular}
\end{tabular}




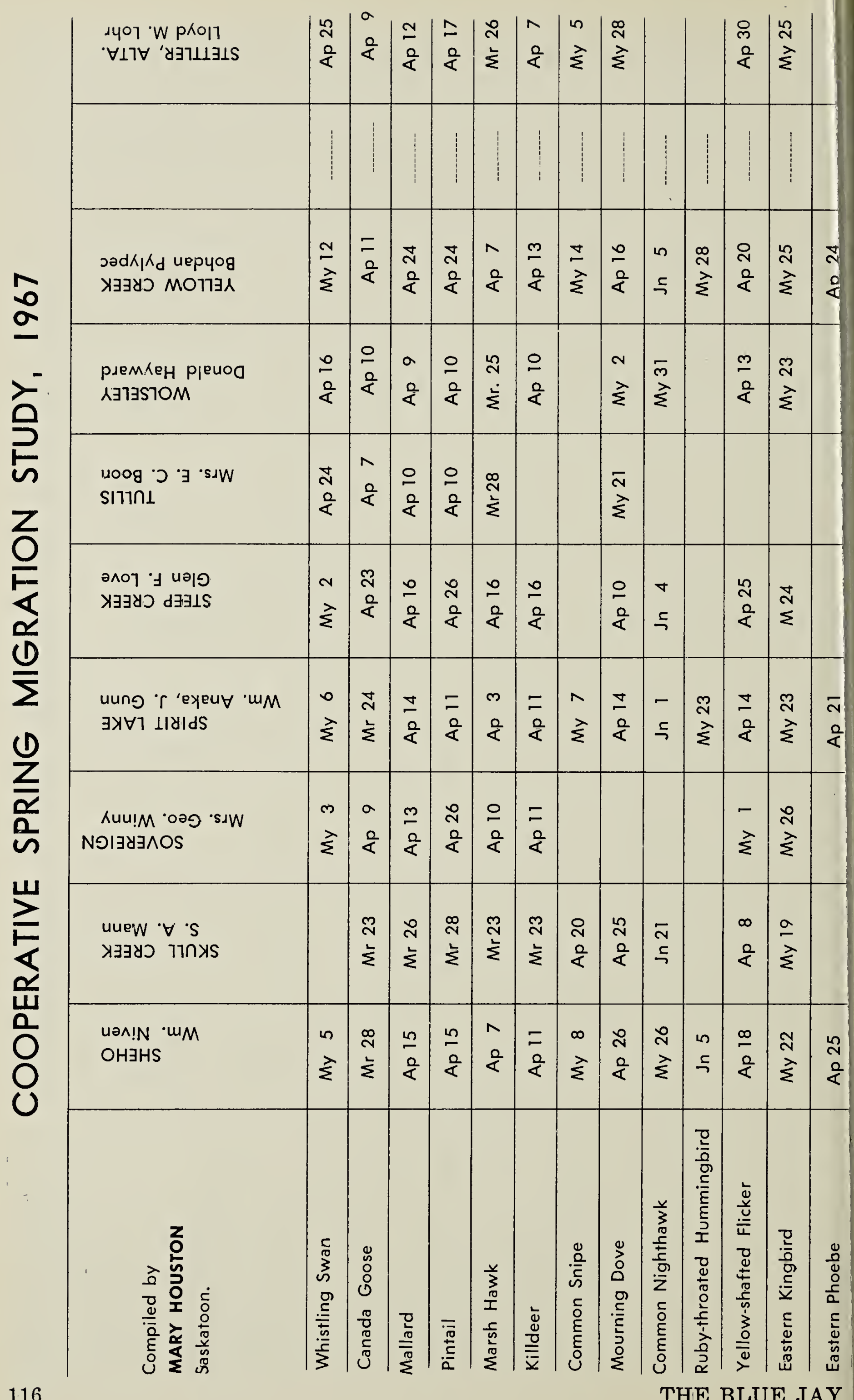


